\title{
Rinometria acústica em crianças submetidas à disjunção maxilar
}

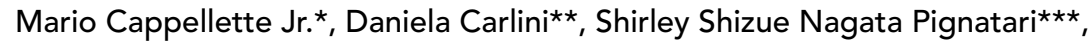 \\ Oswaldo Laércio Mendonça Cruz ${ }^{\star \star \star \star}$, Luc Louis Maurice Weckx $x^{\star \star \star \star}$
}

\section{Resumo}

Objetivo: este estudo foi realizado na Disciplina de Otorrinolaringologia Pediátrica da Escola Paulista de Medicina - UNIFESP, com o objetivo de verificar o comportamento da disjunção maxilar e sua influência na cavidade nasal em crianças de 7 a 8 anos de idade submetidas à rinometria acústica. Metodologia: foram avaliadas 20 crianças respiradoras bucais, portadoras de atresia maxilar diagnosticada clinicamente e submetidas à avaliação objetiva da cavidade nasal pré-disjunção maxilar e pós-disjunção maxilar por meio de rinometria acústica, analisando-se os resultados das áreas transversais mínimas (MCA1 e MCA2) e volumes nasais (VOL1 e VOL2). Resultados: os resultados de MCAl e MCA2 pré-disjunção maxilar apresentaram-se menores que os valores de MCAl e MCA2 pós-disjunção maxilar do lado esquerdo; à direita, a MCAl pré-disjunção maxilar mostrou-se menor que a MCAl pós-disjunção maxilar, aumentos estes estatisticamente significantes, já os VOL1 e VOL2 pré-disjunção foram menores que o VOL1 e VOL2 pós-disjunção maxilar em ambos os lados, ou seja, os resultados desta diferença foram estatisticamente significantes. Conclusão: a rinometria acústica é um método objetivo para avaliar a variação da cavidade nasal. A disjunção maxilar promove um aumento significante da área e volume da cavidade nasal.

Palavras-chave: Atresia maxilar. Disjunção maxilar. Rinometria acústica. Respiração bucal.

\section{INTRODUÇÃO}

O nariz tem papel importante na fisiologia respiratória, promovendo a filtração, aquecimento e umidificação do ar inspirado. O processo respiratório nasal é muito importante, pois a obstrução nasal pode determinar uma respiração bucal suplementar, acarretar a redução da expansão torácica levan- do em longo prazo à alteração do desenvolvimento da caixa torácica, alterações morfológicas da coluna vertebral e do esqueleto facial como a atresia maxilar que, causando alterações oclusais, tem sido cada vez mais observada no dia-a-dia do otorrinolaringologista, do pediatra e tem grande freqüência de aparecimento nas clínicas ortodônticas ${ }^{1,2}$.

\footnotetext{
* Cirurgião-dentista. Especialista em Radiologia pela USF. Mestre em Ortodontia e Ortopedia Facial pela UNICASTELO. Doutor em Ciências pela Escola Paulista de Medicina - UNIFESP e Coordenador do Curso de Especialização em Ortodontia e Ortopedia Facial da ABO-SP e FAMOSP. ** Médica, Mestre e Doutora em Otorrinolaringologia pela Escola Paulista de Medicina - UNIFESP.

*** Médica, Docente e Chefe da Disciplina de Otorrinolaringologia Pediátrica da Escola Paulista de Medicina - UNIFESP. **** Médico, Docente Afiliado da Disciplina de Otorrinolaringologia Pediátrica da Escola Paulista de Medicina - UNIFESP. ***** Médico, Professor Titular do Departamento de Otorrinolaringologia da Escola Paulista de Medicina - UNIFESP.
} 
A atresia maxilar tem como uma das principais causas a respiração bucal de suplência devida à obstrução nasal e/ou por acomodação, existindo ainda outras causas envolvidas na etiologia como: o biotipo facial, problemas de postura mandibular e a perda precoce de dentes.

Acredita-se que a atresia maxilar seja a conseqüência desta série de possíveis alterações, porém observa-se que pode estar relacionada a alterações das dimensões da cavidade nasal, levando então a uma diminuição das áreas transversais mínimas e do volume nasal ${ }^{1,2,3,4}$.

\section{Atresia maxilar}

Em 1996, Normando, Ribeiro, Sotão e Normando ${ }^{4}$ relataram que a respiração bucal, entre outros fatores, está associada à atresia maxilar. A passagem do fluxo de ar pela cavidade nasal produz uma pressão que gera um estímulo ao crescimento maxilar. Na presença de uma respiração bucal, ocorre uma redução nesta pressão e conseqüentemente uma redução ao estímulo de crescimento lateral da maxila. O resultado final é a atresia esquelética da maxila ${ }^{4,5,6}$.

O tratamento da atresia maxilar consiste na expansão do arco maxilar obtido pela disjunção maxilar (que pode ser lenta ou rápida). A expansão rápida, obtida através de forças pesadas, produz um expansão do arco dentário pela abertura da sutura palatina mediana. Acredita-se que a abertura da sutura óssea seja maior quanto menor for a idade do paciente 7,8 . Devido à íntima relação entre as cavidades bucal e nasal, a disjunção maxilar no tratamento da atresia maxilar extrapola os limites da Ortodontia e Ortopedia Facial. Diversos estudos têm tentado comprovar uma relação entre a expansão da maxila e uma diminuição na resistência à entrada de ar pela cavidade nasal. As dimensões laterais da cavidade nasal aumentam em função do afastamento das suas paredes inferior e laterais induzidas pela expansão maxilar ${ }^{2,3,4,7,8,9,10}$.

$\mathrm{Na}$ verdade, a crença que a expansão da maxila seja capaz de produzir uma maior permeabilidade nasal, melhorando os quadros de respiração bucal, vem desde o início do século $\mathrm{XX}^{2,3,4}$, direcionados por um padrão genético já comprometido, sugerese que a "síndrome da face longa" ou "face adenoideana" possa ser agravada pela respiração bucal devido à alta resistência nasal à passagem de ar. As características faciais associadas à respiração bucal incluem uma mandíbula retrognática, incisivos superiores inclinados para a região vestibular, palato ogival, arco maxilar atrésico, lábio superior curto e hipotônico, musculatura peribucal flácida e uma aparência conhecida como facies adenoideano resultante de uma postura constante de lábios entreabertos ${ }^{8,9,10}$. Porém a função nasal respiratória não tem sido adequadamente definida e, portanto, a extensão pela qual a forma dentofacial pode ser influenciada pela forma respiratória não é clara. Há uma dificuldade em quantificar, objetivamente, a obstrução nasal e o modo de respiração.

\section{Rinometria acústica}

O Rinômetro Acústico foi introduzido por Hilberg em $1989^{11}$, com o intuito de estudar melhor os sintomas nasais tão subjetivos, fornecendo informações mais precisas sobre as dimensões da cavidade nasal, auxiliando na quantificação desses sintomas, tornando-os mais objetivos.

É um exame realizado de maneira estática, rápida, não invasiva, que independe do fluxo nasal e que pode ser realizado em qualquer faixa etária $^{11,12}$. Utiliza uma sonda para transmitir e receber o som da fonte eletrônica até o nariz, acoplada a adaptadores narinários, realiza medidas do menor estreitamento da cavidade nasal, dita área transversal mínima (MCA), e estabelece relação desse estreitamento com sua respectiva localização ao longo da cavidade nasal, normalmente fornece dois valores de áreas transversais mínimas, a menor área localizada entre 0 e $22 \mathrm{~mm}$ da narina e a menor área entre 22 e $54 \mathrm{~mm}^{13}$. Como o aparelho fornece medidas da área em $\mathrm{cm}^{2}$ e medida das distâncias destes pontos até a narina em $\mathrm{cm}$, conseguimos obter medidas dos volumes nasais das 
mesmas regiões, V1 (correspondendo ao volume entre 0 e $22 \mathrm{~mm}$ da narina) e V2 (correspondente ao volume entre 22 a $54 \mathrm{~mm}$ da narina), além do Vtotal (correspondente ao volume total da fossa nasal, obtido pela soma de V1 e V2) ${ }^{13}$.

$\mathrm{O}$ equipamento emite uma onda sonora para a cavidade nasal e, realizando medidas com o tempo de emissão e reflexão, fornece a distância dos pontos de reflexão até a narina e, comparando a conformação da onda sonora emitida e a refletida, estabelece a área nesse determinado local, os resultados deste teste são descritos em um gráfico denominado de rinograma (Gráf. 1) ${ }^{13}$.

\section{OBJETIVO}

Estudar a cavidade nasal de 20 pacientes entre 7 e 8 anos de idade, portadores de atresia maxilar diagnosticada por ortodontista, todos os pacientes foram avaliados por exame otorrinolaringológico complementado por avaliação da cavidade nasal por fibra óptica flexível, avaliação ortodôntica e rinometria acústica, tendo como objetivo a observação das variações de abertura maxilar e suas possíveis conseqüências no complexo nasomaxilar e a comparação de valores obtidos com os exames objetivos, tanto pré como pós-disjunção maxilar.

\section{MATERIAL E MÉTODOS}

Foram selecionados 20 pacientes de ambos os gêneros, com idade entre 7 e 8 anos, com dentadura mista e que apresentavam diagnóstico clínico de atresia maxilar. Os pacientes selecionados foram submetidos a: questionário direcionado a pesquisa de sinais e sintomas de obstrução nasal e/ou respiração bucal, exame ortodôntico, exame otorrinolaringológico complementado por exame da cavidade nasal por fibra óptica flexível e avaliação da função respiratória utilizando a rinometria acústica, após a assinatura do termo de consentimento, semelhante ao do projeto de pesquisa já aprovado pelo comitê de Ética em Pesquisa do Hospital São Paulo/ Universidade Federal de São Paulo $\mathrm{N}^{\circ}$ 085/01.

\section{Exame físico ortodôntico}

Consiste na avaliação da assimetria facial e da cavidade bucal que compreende: exame ortodôntico específico, classificação de Angle, ausência de cáries, gengivite, periodontite ou lesões na cavidade bucal e diagnóstico clínico de atresia maxilar

\section{Exame ortodôntico}

Consiste em 5 slides (extrabucais - frontal e perfil do paciente, e intrabucais - frontal, lateral direita e esquerda), avaliação radiográfica (panorâmica e telerradiografia PA e lateral com traçado cefalométrico) e modelos em gesso com o paciente em oclusão.

\section{Ativação e controle de contenção}

A ativação inicial do parafuso tipo Hyrax, consiste de 6 a 8 ativações, o que corresponde respectivamente de uma volta e meia a duas do parafuso expansor e duas ativações diárias, até que se atinja a quantidade de expansão maxilar necessária, que variou de um paciente para outro. Com os objetivos da expansão maxilar atingidos, mantém-se o disjuntor maxilar estabilizado por pelo menos 3 meses para que ocorra a completa neoformação óssea e posterior remoção do aparelho.

\section{Exame físico otorrinolaringológico}

O exame físico compreendeu: inspeção da cavidade bucal e da bucofaringe sob iluminação indireta com o espelho frontal auxiliado por espátula abaixadora de língua, otoscopia realizada com otoscópio e espéculos auriculares e rinoscopia anterior sob iluminação indireta com o espelho frontal e auxílio de espéculo nasal de Cottle, complementado por exame da cavidade nasal por fibra óptica flexível.

\section{Avaliação da cavidade nasal por fibra óptica flexível}

Para complementação do exame físico todos os pacientes foram submetidos a exame da cavidade nasal e rinofaringe por fibra óptica flexível 
em posição sentada, após-aplicação de dois jatos de neotutocaína a $2 \%$ em cada cavidade nasal. O equipamento utilizado foi um endoscópio Machida ${ }^{\circledR}$ ENT - 30PIII de 3,2mm de espessura, acoplado a uma microcâmera Toshiba ${ }^{\circledR}$, com monitor $\mathrm{JVC}^{\circledR}$, fonte de luz Wite Lite - TM e aparelho de vídeocassete Philips Turbo - drive.

Durante a realização do exame adentrou-se inicialmente à cavidade nasal esquerda por sobre a concha nasal inferior e abaixo da concha nasal média, tendo em vista o meato médio, a presença ou não de secreção catarral ou purulenta, pólipos, tumores e alterações anatômicas da região, seguiuse até a coana para observação da tonsila faríngea e suas dimensões - considerando-se como valor normal a ocupação de até $70 \%$ da coana ${ }^{14}$ e ausência de secreção sobre ela. Posteriormente o aparelho foi introduzido paralelamente ao assoalho da cavidade nasal homolateral para observação de presença de secreção, alterações anatômicas, pólipos ou tumores dessa região. Realizou-se exame semelhante na cavidade nasal direita.

- Critérios de inclusão: considerou-se como critérios de inclusão pacientes com 7 a 8 anos de idade, com diagnóstico clínico de atresia maxilar realizado por ortodontista, que valorizou as características faciais associadas à respiração bucal.

- Critérios de exclusão: hipertrofia de conchas nasais ocupando totalmente a cavidade nasal; alterações anatômicas do septo nasal; presença de tumores ou pólipos nasais; presença de tonsila faríngea ocupando mais de $70 \%$ das coanas ${ }^{14}$; presença de secreção purulenta em meatos médios ou assoalho das cavidades nasais, caracterizando quadro infeccioso.

\section{Rinometria acústica}

Todos os pacientes selecionados foram submetidos ao exame de Rinometria Acústica, realizado na Disciplina de Otorrinolaringologia Pediátrica. Todas as medidas foram realizadas com o paciente sentado, pós-uso de vasoconstritor tópico (cloridrato de oximetazolina $0,5 \mathrm{mg} / \mathrm{dl}$ ) após uma es-

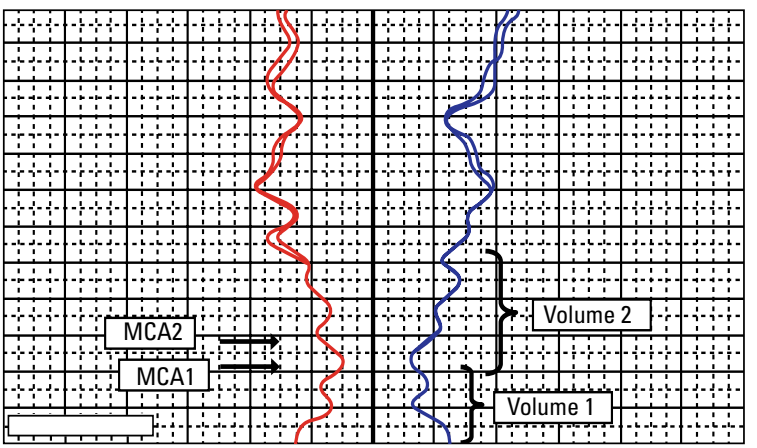

GRÁFICO 1 - MCA1 (menor área entre 0 e 22mm da narina), MCA2 (menor área entre 22 e $54 \mathrm{~mm}$ da narina), Volume nasal 1 (entre 0 e $22 \mathrm{~mm}$ da narina) e Volume nasal 2 (entre 22 e $54 \mathrm{~mm}$ da narina).

pera mínima de 20 minutos em sala climatizada à temperatura de $20^{\circ}$ a $22^{\circ}$ Celsius, utilizandose para isso um ar condicionado Springer 7500 - Mundial, com umidade relativa do ar entre 40\% e 50\%, como o indicado na Padronização da Rinometria Acústica, seguindo-se as recomendações do Comitê Internacional de Normatização da Rinometria e Rinomanometria de $1998^{13}$.

Foram realizadas de 2 a 3 aferições em cada narina, pré-disjunção e após o término da disjunção, porém como a reprodutibilidade do exame é grande, apenas um gráfico de cada exame foi escolhido e analisado ${ }^{12,15}$. Os gráficos obtidos foram avaliados de acordo com a observação de duas medidas de área de cada cavidade e duas medidas de volume de cada cavidade respectivamente: $\mathrm{MCAl}$ (menor área entre 0 e $22 \mathrm{~mm}$ da narina), MCA2 (menor área entre 22 e $54 \mathrm{~mm}$ da narina), Vl (volume entre 0 e $22 \mathrm{~mm}$ da narina) e V2 (volume entre 22 e $54 \mathrm{~mm}$ da narina).

\section{Método estatístico}

Dada a natureza das variáveis previamente definidas, foram empregados testes não paramétricos.

Para avaliação das variáveis quanto ao seu comportamento em relação ao pré e pós-uso de disjuntor maxilar, foi empregado o teste para grupos não independentes de Wilcoxon. Para verificar correlação entre as variáveis utilizou-se o teste de Spearman. 
Em todos os casos o nivel de rejeição para a hipótese de nulidade foi fixado sempre em um valor igual ou menor do que 0,05 (5\%).

\section{RESULTADOS}

De acordo com os exames de rinometria acústica obteve-se como dados os valores das áreas transversais mínimas entre 0 e $22 \mathrm{~mm}$ da narina, dita MCAl, valores da áreas transversais mínimas entre 22 e $54 \mathrm{~mm}$ da narina, dita MCA2, e os respectivos volumes nasais 1 e 2 , todos estes relacionados nas tabelas 1, 2, 3 e 4 .

\section{DISCUSSÃO}

De acordo com a literatura, a maioria dos estudos tenta relacionar a disjunção maxilar com a ca- vidade nasal, utilizando para isso a rinomanometria computadorizada ${ }^{2,3,4}$, ou seja verificando mudanças na resistência nasal do indivíduo após a disjunção maxilar. Neste estudo procurou-se avaliar as variações da cavidade nasal pré e pós-disjunção maxilar utilizando a rinometria acústica, que fornece áreas transversais mínimas e volumes nasais.

De acordo com os resultados observados nas tabelas 1 e 2, quando comparados os resultados pré e pós-disjunção maxilar pelo teste de Wilcoxon, os valores das MCA1 e MCA2 do lado esquerdo e MCAl do lado direito pré-disjunção maxilar apresentaram-se menores do que as mesmas áreas pós-disjunção maxilar e que estes resultados foram estatisticamente significante, interpreta-se então que a cavidade nasal apresentou um aumento das

Tabela 1 - Medidas das MCA1, MCA2, VOL1 e VOL2 de cavidade nasal direita pré e pós-disjunção maxilar, análise estatística segundo método de Wilcoxon e respectivos resultados estatísticos.

\begin{tabular}{|c|c|c|c|c|c|c|c|c|c|c|}
\hline \multicolumn{11}{|c|}{ Cavidade nasal direita } \\
\hline Número & Gênero & Idade & MCA1 pré & MCA1 pós & MCA2 pré & MCA2 pós & VOL1 pré & VOL1 pós & VOL2 pré & VOL2 pós \\
\hline 1 & $\mathrm{~F}$ & 8 & 0,31 & 0,46 & 0,92 & 1,26 & 1,31 & 1,52 & 4,48 & 6,13 \\
\hline 2 & M & 8 & 0,21 & 0,32 & 0,21 & 0,36 & 1,20 & 1,23 & 1,50 & 4,34 \\
\hline 3 & $\mathrm{~F}$ & 8 & 0,44 & 0,45 & 0,79 & 0,78 & 1,47 & 1,75 & 4,08 & 3,04 \\
\hline 4 & M & 8 & 0,43 & 0,37 & 0,41 & 0,46 & 1,44 & 1,12 & 2,99 & 3,29 \\
\hline 5 & M & 8 & 0,34 & 0,46 & 0,37 & 1,05 & 1,06 & 1,47 & 2,41 & 4,27 \\
\hline 6 & M & 8 & 0,23 & 0,25 & 0,40 & 0,26 & 1,03 & 1,02 & 2,50 & 4,55 \\
\hline 7 & M & 8 & 0,32 & 0,30 & 0,64 & 0,72 & 1,16 & 1,18 & 2,91 & 3,19 \\
\hline 8 & $F$ & 8 & 0,30 & 0,43 & 0,83 & 1,08 & 1,18 & 1,44 & 3,19 & 4,40 \\
\hline 9 & $M$ & 8 & 0,20 & 0,18 & 0,34 & 0,18 & 1,18 & 1,07 & 1,59 & 1,34 \\
\hline 10 & $M$ & 8 & 0,32 & 0,35 & 0,84 & 0,62 & 1,36 & 1,37 & 3,99 & 4,32 \\
\hline 11 & $M$ & 7 & 0,28 & 0,37 & 0,57 & 0,57 & 1.08 & 1,08 & 2,67 & 2,67 \\
\hline 12 & $M$ & 7 & 0,35 & 0,39 & 0,45 & 0,45 & 1,13 & 1,30 & 2,69 & 2,64 \\
\hline 13 & $F$ & 7 & 0,23 & 0,44 & 0,16 & 0,58 & 1,12 & 1,39 & 1,31 & 2,75 \\
\hline 14 & $\mathrm{~F}$ & 7 & 0,32 & 0,27 & 0,67 & 0,46 & 1,03 & 1,03 & 2,79 & 2,79 \\
\hline 15 & $M$ & 7 & 0,53 & 0,49 & 0,86 & 0,86 & 1,66 & 1,57 & 4,33 & 4,00 \\
\hline 16 & $F$ & 7 & 0,23 & 0,34 & 0,24 & 0,45 & 1,20 & 1,20 & 2,21 & 2,82 \\
\hline 17 & $M$ & 7 & 0,55 & 0,46 & 0,77 & 0,79 & 1,62 & 1,73 & 4,35 & 4,35 \\
\hline 18 & $F$ & 7 & 0,19 & 0,27 & 0,49 & 0,64 & 1,04 & 1,15 & 2,32 & 2,96 \\
\hline 19 & $F$ & 7 & 0,33 & 0,43 & 0,40 & 0,59 & 1,23 & 1,37 & 3,03 & 3,37 \\
\hline 20 & $\mathrm{~F}$ & 7 & 0,29 & 0,35 & 0,43 & 0,41 & 1,27 & 1,17 & 2,17 & 2,03 \\
\hline \multicolumn{3}{|c|}{ Método de Wilcoxon } & \multicolumn{2}{|c|}{$P=0,20 *$} & \multicolumn{2}{|c|}{$P=0,107$ ( n.s.) } & \multicolumn{2}{|c|}{$P=0,058$ (n. s.) } & \multicolumn{2}{|c|}{$P=0,012 *$} \\
\hline
\end{tabular}

${ }^{*}$ Considerando $p \leq 0,05(5 \%)$ como significativo e $p>0,05(5 \%)$ como não significativo (n.s.). 
Tabela 2 - Medidas das MCA1 , MCA2, VOL1 e VOL2 de cavidade nasal esquerda pré e pós-disjunção maxilar, análise estatística segundo método de Wilcoxon e respectivos resultados estatísticos.

\begin{tabular}{|c|c|c|c|c|c|c|c|c|c|c|}
\hline \multicolumn{11}{|c|}{ Cavidade nasal esquerda } \\
\hline Número & Gênero & Idade & MCA1 pré & MCA1 pós & MCA2 pré & MCA2 pós & VOL1 pré & VOL1 pós & V0L2 pré & VOL2 pós \\
\hline 1 & $\mathrm{~F}$ & 8 & 0,46 & 0,50 & 0,89 & 0,95 & 1,31 & 1,47 & 5,15 & 4,07 \\
\hline 2 & M & 8 & 0,21 & 0,39 & 0,19 & 0,39 & 1,17 & 1,28 & 0,91 & 4,07 \\
\hline 3 & $\mathrm{~F}$ & 8 & 0,41 & 0,48 & 0,50 & 0,95 & 1,24 & 1,51 & 3,52 & 4,06 \\
\hline 4 & $\mathrm{M}$ & 8 & 0,43 & 0,49 & 0,49 & 0,56 & 1,43 & 1,38 & 3,19 & 3,82 \\
\hline 5 & M & 8 & 0,34 & 0,48 & 0,68 & 1,00 & 1,14 & 1,55 & 2,52 & 4,47 \\
\hline 6 & M & 8 & 0,43 & 0,47 & 0,50 & 0,61 & 1,19 & 1,31 & 2,86 & 3,53 \\
\hline 7 & M & 8 & 0,31 & 0,32 & 0,53 & 0,65 & 1,13 & 1,15 & 2,63 & 3,00 \\
\hline 8 & $\mathrm{~F}$ & 8 & 0,43 & 0,49 & 0,61 & 1,13 & 1,42 & 1,49 & 3,44 & 4,28 \\
\hline 9 & M & 8 & 0,34 & 0,35 & 0,57 & 0,44 & 1,17 & 1,31 & 2,32 & 2,03 \\
\hline 10 & M & 8 & 0,34 & 0,46 & 0,62 & 0,88 & 1,47 & 1,61 & 4,11 & 5,18 \\
\hline 11 & M & 7 & 0,40 & 0,34 & 0,53 & 0,47 & 1,12 & $1,09 i$ & 2,50 & 2,30 \\
\hline 12 & M & 7 & 0,33 & 0,30 & 0,60 & 0,47 & 1,14 & 1,18 & 2,74 & 2,53 \\
\hline 13 & $\mathrm{~F}$ & 7 & 0,41 & 0,43 & 0,46 & 0,65 & 1,19 & 1,37 & 3,81 & 3,33 \\
\hline 14 & $\mathrm{~F}$ & 7 & 0,32 & 0,36 & 0,58 & 0,55 & 1,05 & 1,19 & 2,02 & 3,77 \\
\hline 15 & M & 7 & 0,43 & 0,41 & 0,68 & 0,60 & 1,36 & 1,39 & 2,82 & 2,79 \\
\hline 16 & $\mathrm{~F}$ & 7 & 0,37 & 0,39 & 0,40 & 0,57 & 1,31 & 1,19 & 2,78 & 3,06 \\
\hline 17 & $M$ & 7 & 0,52 & 0,43 & 0,84 & 0,92 & 1,57 & 1,79 & 3,92 & 4,77 \\
\hline 18 & $\mathrm{~F}$ & 7 & 0,27 & 0,31 & 0,60 & 0,57 & 1,05 & 1,29 & 2,25 & 2,96 \\
\hline 19 & $F$ & 7 & 0,44 & 0,48 & 0,51 & 0,66 & 1,30 & 1,33 & 3,28 & 3,56 \\
\hline 20 & $\mathrm{~F}$ & 7 & 0,35 & 0,59 & 0,34 & 0,73 & 1,13 & 1,69 & 1,49 & 3,82 \\
\hline \multicolumn{3}{|c|}{ Método de Wilcoxon } & \multicolumn{2}{|c|}{$P=0,014$ * } & \multicolumn{2}{|c|}{$P=0,008^{*}$} & \multicolumn{2}{|c|}{$P=0,001^{*}$} & \multicolumn{2}{|c|}{$P=0,010^{*}$} \\
\hline
\end{tabular}

${ }^{*}$ Considerando $p \leq 0,05(5 \%)$ como significativo e $p>0,05(5 \%)$ como não signific ativo (n.s.).

áreas, tanto a esquerda como a direita, provavelmente secundárias à correção da atresia maxilar, ou seja pós-disjunção maxilar, com exceção da MCA2 do lado direito que não apresentou uma variação estatisticamente significante, apesar de grande parte dos pacientes apresentarem valores de áreas maiores após a disjunção, credita-se esta não significância ao tamanho da amostra. No caso dos volumes nasais observa-se que o VOL1 e o VOL2 da cavidade nasal esquerda e o VOL2 da cavidade nasal direita pré-disjunção maxilar mostraram-se menores que as mesmas áreas após a disjunção maxilar, sendo este resultado estatisticamente significante, sugerindo então que a disjunção maxilar pode causar variações dentro da cavidade nasal, porém o VOL1 do lado direi- to pré-disjunção não mostrou variação estatisticamente significante quando comparado com o VOL1 pós-disjunção maxilar, mas quando observa-se os valores individuais de cada paciente para o VOLl pré e compara-se com o VOL1 pós-disjunção maxilar nota-se que estes valores aumentaram, portanto este comportamento poderia estar ocorrendo devido ao tamanho da amostra ser reduzida.

Foram feitos então testes para verificar se as variações encontradas nas áreas (MCA1 e MCA2) estariam relacionadas às variações de volumes (VOL1 e VOL2) tanto pré-disjunção maxilar quanto pós-disjunção e, de acordo com o teste de Spearman, pode-se observar que MCAl pré e VOL1 pré, MCA2 pré e VOL2 pré, MCAl pós 
Tabela 3 - MCA1, MCA2, VOL1 e VOL2, à direita, pré e pós-disjunção maxilar, análise estatística dos dados de acordo com o teste de Spearman e resultados estatísticos.

\begin{tabular}{|c|c|c|c|c|c|c|c|c|c|c|}
\hline \multirow{2}{*}{$\frac{\text { Número }}{1}$} & \multirow{2}{*}{$\frac{\text { Gênero }}{\mathrm{F}}$} & \multirow{2}{*}{$\begin{array}{c}\text { Idade } \\
8\end{array}$} & \multicolumn{2}{|c|}{ MCA1 pré X V0L1 pré } & \multicolumn{2}{|c|}{ MCA2 pré X V0L2 pré } & \multicolumn{2}{|c|}{ MCA1 pós X V0L1 pós } & \multicolumn{2}{|c|}{ MCA2 pós X VOL2 pós } \\
\hline & & & 0,31 & 1,31 & 0,92 & 4,48 & 0,46 & 1,52 & 1,26 & 6,13 \\
\hline 2 & M & 8 & 0,21 & 1,20 & 0,21 & 1,50 & 0,32 & 1,23 & 0,36 & 4,34 \\
\hline 3 & $\mathrm{~F}$ & 8 & 0,44 & 1,47 & 0,79 & 4,08 & 0,45 & 1,75 & 0,78 & 3,04 \\
\hline 4 & M & 8 & 0,43 & 1,44 & 0,41 & 2,99 & 0,37 & 1,12 & 0,46 & 3,29 \\
\hline 5 & M & 8 & 0,34 & 1,06 & 0,37 & 2,41 & 0,46 & 1,47 & 1,05 & 4,27 \\
\hline 6 & M & 8 & 0,23 & 1,03 & 0,40 & 2,50 & 0,25 & 1,02 & 0,26 & 4,55 \\
\hline 7 & $M$ & 8 & 0,32 & 1,16 & 0,64 & 2,91 & 0,30 & 1,18 & 0,72 & 3,19 \\
\hline 8 & $\mathrm{~F}$ & 8 & 0,30 & 1,18 & 0,83 & 3,19 & 0,43 & 1,44 & 1,08 & 4,40 \\
\hline 9 & M & 8 & 0,20 & 1,18 & 0,34 & 1,59 & 0,18 & 1,07 & 0,18 & 1,34 \\
\hline 10 & M & 8 & 0,32 & 1,36 & 0,84 & 3,99 & 0,35 & 1,37 & 0,62 & 4,32 \\
\hline 11 & M & 7 & 0,28 & 1.08 & 0,57 & 2,67 & 0,37 & 1,08 & 0,57 & 2,67 \\
\hline 12 & $M$ & 7 & 0,35 & 1,13 & 0,45 & 2,69 & 0,39 & 1,30 & 0,45 & 2,64 \\
\hline 13 & $\mathrm{~F}$ & 7 & 0,23 & 1,12 & 0,16 & 1,31 & 0,44 & 1,39 & 0,58 & 2,75 \\
\hline 14 & $\mathrm{~F}$ & 7 & 0,32 & 1,03 & 0,67 & 2,79 & 0,27 & 1,03 & 0,46 & 2,79 \\
\hline 15 & $M$ & 7 & 0,53 & 1,66 & 0,86 & 4,33 & 0,49 & 1,57 & 0,86 & 4,00 \\
\hline 16 & $\mathrm{~F}$ & 7 & 0,23 & 1,20 & 0,24 & 2,21 & 0,34 & 1,20 & 0,45 & 2,82 \\
\hline 17 & $M$ & 7 & 0,55 & 1,62 & 0,77 & 4,35 & 0,46 & 1,73 & 0,79 & 4,35 \\
\hline 18 & $\mathrm{~F}$ & 7 & 0,19 & 1,04 & 0,49 & 2,32 & 0,27 & 1,15 & 0,64 & 2,96 \\
\hline 19 & $\mathrm{~F}$ & 7 & 0,33 & 1,23 & 0,40 & 3,03 & 0,43 & 1,37 & 0,59 & 3,37 \\
\hline 20 & $\mathrm{~F}$ & 7 & 0,29 & 1,27 & 0,43 & 2,17 & 0,35 & 1,17 & 0,41 & 2,03 \\
\hline
\end{tabular}

* Considerando $p \leq 0,050(5 \%)$ como significativo e $p>0,05(5 \%)$ como não significativo.

e VOL1 pós, MCA2 pós e VOL2 pós, à direita, estão relacionados entre si e esta relação é estatisticamente significante, ou seja, quando ocorre uma variação na área, imediatamente esta variação também é encontrada no volume nasal da cavidade nasal direita. À esquerda observamos o mesmo comportamento com exceção da MCA2 pré e VOL2 pré-disjunção maxilar, que não apresentou variação estatisticamente significante. Com isso podemos sugerir que quando realizamos a rinometria acústica para avaliar a disjunção maxilar, ou ainda variação da cavidade nasal, podemos coletar apenas os resultados da variação de volume nasal, pois tanto áreas transversais como volumes nasais pré e pós-disjunção maxilar estão relacionados entre si, para este grupo de pacientes.

\section{CONCLUSÃO}

Com este estudo podemos sugerir que a rinometria acústica, para este grupo de pacientes foi, de acordo com a literatura ${ }^{15}$, um método objetivo de avaliação da variação da cavidade nasal frente à disjunção maxilar e que a disjunção maxilar demonstrou aumentar as áreas e volumes nasais neste grupo de pacientes portadores de atresia maxilar. 
Tabela 4 - MCA1, MCA2, VOL1 e VOL2, à esquerda, pré e pós-disjunção maxilar, análise estatísitica dos dados de acordo com 0 teste de Spearman e resultados estatísticos.

\begin{tabular}{|c|c|c|c|c|c|c|c|c|c|c|}
\hline \multirow{2}{*}{$\frac{\text { Número }}{1}$} & \multirow{2}{*}{$\frac{\text { Gênero }}{F}$} & \multirow{2}{*}{$\begin{array}{c}\text { Idade } \\
8\end{array}$} & \multicolumn{2}{|c|}{ MCA1 pré X V0L1 pré } & \multicolumn{2}{|c|}{ MCA2 pré X VOL 2 pré } & \multicolumn{2}{|c|}{ MCA1 pós X V0L1 pós } & \multicolumn{2}{|c|}{ MCA2 pós X VOL2 pós } \\
\hline & & & 0,46 & 1,31 & 0,89 & 5,15 & 0,50 & 1,47 & 0,95 & 4,07 \\
\hline 2 & $M$ & 8 & 0,21 & 1,17 & 0,19 & 0,91 & 0,39 & 1,28 & 0,39 & 4,07 \\
\hline 3 & $\mathrm{~F}$ & 8 & 0,41 & 1,24 & 0,50 & 3,52 & 0,48 & 1,51 & 0,95 & 4,06 \\
\hline 4 & M & 8 & 0,43 & 1,43 & 0,49 & 3,19 & 0,49 & 1,38 & 0,56 & 3,82 \\
\hline 5 & $M$ & 8 & 0,34 & 1,14 & 0,68 & 2,52 & 0,48 & 1,55 & 1,00 & 4,47 \\
\hline 6 & $M$ & 8 & 0,43 & 1,19 & 0,50 & 2,86 & 0,47 & 1,31 & 0,61 & 3,53 \\
\hline 7 & $M$ & 8 & 0,31 & 1,13 & 0,53 & 2,63 & 0,32 & 1,15 & 0,65 & 3,00 \\
\hline 8 & $\mathrm{~F}$ & 8 & 0,43 & 1,42 & 0,61 & 3,44 & 0,49 & 1,49 & 1,13 & 4,28 \\
\hline 9 & $M$ & 8 & 0,34 & 1,17 & 0,57 & 2,32 & 0,35 & 1,31 & 0,44 & 2,03 \\
\hline 10 & $M$ & 8 & 0,34 & 1,47 & 0,62 & 4,11 & 0,46 & 1,61 & 0,88 & 5,18 \\
\hline 11 & $M$ & 7 & 0,40 & 1,12 & 0,53 & 2,50 & 0,34 & $1,09 i$ & 0,47 & 2,30 \\
\hline 12 & $M$ & 7 & 0,33 & 1,14 & 0,60 & 2,74 & 0,30 & 1,18 & 0,47 & 2,53 \\
\hline 13 & $\mathrm{~F}$ & 7 & 0,41 & 1,19 & 0,46 & 3,81 & 0,43 & 1,37 & 0,65 & 3,33 \\
\hline 14 & $\mathrm{~F}$ & 7 & 0,32 & 1,05 & 0,58 & 2,02 & 0,36 & 1,19 & 0,55 & 3,77 \\
\hline 15 & $M$ & 7 & 0,43 & 1,36 & 0,68 & 2,82 & 0,41 & 1,39 & 0,60 & 2,79 \\
\hline 16 & $\mathrm{~F}$ & 7 & 0,37 & 1,31 & 0,40 & 2,78 & 0,39 & 1,19 & 0,57 & 3,06 \\
\hline 17 & $M$ & 7 & 0,52 & 1,57 & 0,84 & 3,92 & 0,43 & 1,79 & 0,92 & 4,77 \\
\hline 18 & $\mathrm{~F}$ & 7 & 0,27 & 1,05 & 0,60 & 2,25 & 0,31 & 1,29 & 0,57 & 2,96 \\
\hline 19 & $\mathrm{~F}$ & 7 & 0,44 & 1,30 & 0,51 & 3,28 & 0,48 & 1,33 & 0,66 & 3,56 \\
\hline 20 & $\mathrm{~F}$ & 7 & 0,35 & 1,13 & 0,34 & 1,49 & 0,59 & 1,69 & 0,73 & 3,82 \\
\hline \multicolumn{3}{|c|}{ Teste de Spearman } & \multicolumn{2}{|c|}{$P=0,001^{*}$} & \multicolumn{2}{|c|}{$P=0,113$ (n.s.) } & \multicolumn{2}{|c|}{$P=0,000^{*}$} & \multicolumn{2}{|c|}{$P=0,001^{*}$} \\
\hline
\end{tabular}

* Considerando $p \leq 0,050(5 \%)$ como significativo e $p>0,05(5 \%)$ como não significativo.

\title{
Acoustic rhinometry in children undergoing a rapid maxillary expansion
}

\begin{abstract}
Aim: this research study was carried out by professionals of the Paulista School of Medicine - UNIFESP and it relates to the Pediatric Otorhinolaryngology subject. The current study aimed at checking the behaviour of the maxillary dysjunction and its influence in the nasal cavity of children between 7 and 8 years of age who underwent acoustic rhinometry. Methods: 20 mouth-breathing subjects presenting maxillary atresia diagnosed clinically were evaluated. Subjects underwent objective evaluation of the nasal cavity before and after maxillary dysjunction by means of acoustic rhinometry. Results of the minimal transverse area (MCA1) and the nasal mass (VOL1 and VOL2) were analyzed. Results: in the left side, MCA1 and MCA2 results before maxillary dysjunction were smaller than MCA1 and MCA2 after maxillary dysjunction. In the right side, MCA1 before maxillary dysjunction was smaller than MCA1 after maxillary dysjunction. Such increaments showed to be statistically significant. Conclusion: acoustic rhinometry is an objective method of evaluating the variation of the nasal cavity. Maxillary dysjunction causes a significant increase in the nasal cavity area and volume.
\end{abstract}

Key words: Maxillary atresia. Rapid maxillary expansion. Acoustic rhinometry. Mouth breathing. 


\section{REFERÊNCIAS}

1. HAAS, A. J. Rapid expansion of the maxillary dental arch and nasal cavity by opening the midpalatal suture. Angle Orthod Appleton, v. 31, no. 2, p. 73-89, 1961

2. HARTGERINK, D. V.; VIG, P. S.; ABBOTT, D. W. The effect os rapid maxillary expansion on nasal airway resistence. Am J Orthod Dentofacial Orthop, St. Louis, v. 92, no. 5, p. 381-388, 1987.

3. LINDER-ARONSON, S.; ASCHAN, G. Nasal resistance to breathing and palatal height before and after expansion of the median palatine suture. Odontol Revy, Lund, v. 14, no. 3 , p. $254-270,1963$.

4. NORMANDO, A. D. C.; RIBEIRO, K. C. F.; SOTÃO, A. B.; NORMANDO, V. M. F. Os efeitos da expansão rápida da maxila sobre o fluxo aéreo nasal: revisão da literatura e apresentação de um caso clínico. Rev Paraense de Odont, Belem, v. 1, n. 2, p. 19-26, 1996

5. MOSS, J. P. Rapid expansion of the maxillary arch. Part I. J Pract Orthod, Hempstead, v. 2, no. 5, p. 165-171, 1968

6. MOSS, J. P. Rapid expansion of the maxillary arch. Part II. J Pract Orthod, Hempstead, v. 2, no. 5, p. 215-223, 1968

7. MAZZIERO, E. T.; HENRIQUES, J. F. C.; FREITAS, M. R. Estudo cefalométrico em norma frontal das alterações dentoesqueléticas após a expansão rápida da maxila. Ortodontia, São Paulo, v. 29, n. 1, p. 31-40, 1996.

8. TIMMS, D. J. Some medical aspects of rapid maxillary expancion. Br J Orthod, London, v. 1, no. 4, p. 127-132, July, 1974.
9. WARREN, D. W.; HERSHEY, H. G.; TURVEY, T. A.; HINTON, V. A.; HAIRFIELD, W. M. The nasal airway following maxillary expansion. Am J Orthod Dentofacial Orthop, St. Louis, v. 91, no. 2, p. 111-116, 1987.

10. WHITE, B. C.; WOODSIDE, D. G.; COLE, P. The effect of rapid maxillary expansion on nasal airway resistence. J Otolaryngol, Hamilton, v. 18, no. 4, p. 137-143, June 1989.

11. HILBERG, O.; JACKSON, A. C.: SWIFT, D. L.; PEDERSEN, O F. Acoustic rhinometry: evaluation of nasal cavity geometry by acoustic reflection. J Appl Physiol, Washington, v. 66, no. 1, p. 295-303, 1989.

12. GRYMER, L. F.; HILBERG, O.; PEDERSEN, O. F. Prediction of nasal obstruction based on clinical examination and acoustic rhinometry. Rhinology, Leiden, v. 35, p. 53-57, 1997

13. COMITEE ON STANDARDIZATION OF ACOUSTIC RHINOMETRY. Recommendations for technical especifications and standar operating procedures, European rhinologic society. In: INTERNATIONAL SYMPOSIUM OF INFECTION AND ALLERGY OF THE NOSE. MEETING. Abstracts...Viena: [s.n.] 1998.

14. CHAMI, F. A. I. Avaliação nasofibroscópica e radiológica de pacientes com hiperplasia da amígdala faríngea. 1997. Dissertação ( Mestrado)-Escola Paulista de Medicina, Universidade Federal de São Paulo, São Paulo, 1997.

15. ROITHMANN, R.; COLE, P.; CHAPNIK, J.; BARRETO, S. M SZALAI, J. P.; ZAMEL, N. Acoustic rhinometry, rhinomanometry, and the sensation of nasal patency: a correlative study. J Otolaryngol, Hamilton, v. 23 , no. 6, p. 454-458, 1994

\section{Endereço de correspondência}

Mario Cappellette Jr.

Rua Salete $n^{\circ} 200,10^{\circ}$ andar

CEP: 02.016-001 São Paulo/SP

E-mail: mjrcappellette@terra.com.br 